TAIWANESE JOURNAL OF MATHEMATICS

Vol. 10, No. 1, pp. 233-241, January 2006

This paper is available online at http://www.math.nthu.edu.tw/tjm/

\title{
ON THE BANACH-STONE PROBLEM FOR $L^{p}$-SPACES
}

\author{
Chun-Yen Chou, Wu-Lang Day and Jyh-Shyang Jeang
}

\begin{abstract}
The Banach-Stone problem for $L^{p}$-spaces is to assert when a linear isometry between $L^{p}$-spaces is a weighted composition operator. We shall show that every $\sigma$-finite measure space with Sikorski's property solves the Banach-Stone probelm. In addition, we show that if $X$ is a totally ordered and Dedekind complete, then every $\sigma$-finite $\mu$-separable measure space $(X, \mathcal{B}, \mu)$ has Sikorski's property.
\end{abstract}

\section{INTRODUCTION}

Let $X$ and $Y$ be locally compact Hausdorff spaces. A classical Banach-Stone Theorem (see, e.g., Behrends [2, p. 138]) states that every isometry $T$ from the Banach space $C_{0}(X)$ of continuous functions vanishing at infinity onto another $C_{0}(Y)$ is a weighted composition operator $T f=h \cdot(f \circ \varphi)$, for all $f$ in $C_{0}(X)$. In this paper, we call such a map $T$ a $B S$ map. We ask similar questions for operators between $L^{p}$-spaces. Given two measure spaces $(X, \mathcal{B}, \mu)$ and $(Y, \mathcal{A}, \nu)$, is every linear isometry $T: L^{p}(X) \rightarrow L^{q}(Y)$ a BS map?

In [1] (see also [13, p. 415]), an affirmative answer is given for linear isometries $T: L^{p}[0,1] \rightarrow L^{p}[0,1](1 \leq p<\infty, p \neq 2)$. In [10], Lamperti showed that, for $(X, \mathcal{B}, \mu)$ a $\sigma$-finite measure space, every linear isometry $T: L^{p}(X) \rightarrow L^{p}(X)$ $(1 \leq p<\infty, p \neq 2)$ is given by a still simple form $T \chi_{B}=h \cdot \chi_{\Phi(B)}$ for all $B$ in $\mathcal{B}$. We call such a map $T$ a Lamperti map. Note that, in [10], it is not said if $T$ is a BS map or not, and this is still unknown for the time being.

Let $(X, \mathcal{B}, \mu)$ and $(Y, \mathcal{A}, \nu)$ be measure spaces and $1 \leq p, q \leq \infty$. A map $T: L^{p}(X) \rightarrow L^{q}(Y)$ is called disjointness preserving if $f \cdot g=0$ a.e. $[\mu]$ implies $T f \cdot T g=0$ a.e. $[\nu]$ for all $f, g$ in $L^{p}(X)$. We shall see that every (surjective when $p=q=\infty$ ) linear isometry $T: L^{p}(X) \rightarrow L^{q}(Y)$ (either $1 \leq p, q<\infty, p \neq$

Received April 11, 2005.

Communicated by Ngai-Ching Wong.

2000 Mathematics Subject Classification: Primary 46E30, Secondary 46S20, 47A65.

Key words and phrases: Banach-Stone theorem, Disjointness preserving operator.

Partially supported by Taiwan National Science Council under grant: NSC 93-2115-M-145-001. 
$2, q \neq 2$ or $p=q=\infty$, we will call such an pair $(p, q)$ accessible $)$ is disjointness preserving. Therefore, it suffices to study merely bounded disjointness preserving operators.

We shall prove that (i) every $\sigma$-finite measure space $(X, \mathcal{B}, \mu)$ solves the Lamperti problem for $L^{p}$-spaces, that is, for an arbitrary measure space $(Y, \mathcal{A}, \nu)$ and an accessible $(p, q)$, every (surjective when $p=q=\infty$ ) bounded disjointness preserving operator $T: L^{p}(X) \rightarrow L^{q}(Y)$ is a Lamperti map; (ii) every $\sigma$-finite measure space $(X, \mathcal{B}, \mu)$ with Sikorski's property solves the Banach-Stone problem for $L^{p}$-spaces, that is, for an arbitrary measure space $(Y, \mathcal{A}, \nu)$ and an accessible $(p, q)$, every (surjective when $p=q=\infty$ ) bounded disjointness preserving operator $T: L^{p}(X) \rightarrow L^{q}(Y)$ is a BS map. Note that we have included the case $p=q=\infty$ here.

In [11], Lessard used a topological approach with some technical lifting theorems to give the result: Every Lamperti map $T: L^{p}(X, \mathcal{B}, \mu) \rightarrow L^{p}(Y, \mathcal{A}, \nu)$ is a BS map, if $\mu$ is tight. A finite Baire measure $\mu$ on a topological space $X$ is said to be tight if for every $\epsilon>0$, there exists a compact set $K$ in $X$ such that $\mu^{*}(K)>\mu(X)-\epsilon$, where $\mu^{*}$ denotes the outer measure determined by $\mu$.

We shall use an order theoretical approach to give a different sufficient condition (see Proposition 8) for a measure space $(X, \mathcal{B}, \mu)$ solving the Banach-Stone problem for $L^{p}$-spaces. We note that $\left(\mathbb{N}, 2^{\mathbb{N}}, \mu\right)$ is, in general, not tight and thus Lessard's theorem does not apply. However, Proposition 8 below does help. And as wellknown examples, $\mathbb{R}^{n}$ with Borel measure and Hilbert cube with an appropriate measure, satisfy our conditions and solve the Banach-Stone problem.

\section{The LAmperti Problem}

Let $(X, \mathcal{B}, \mu)$ and $(Y, \mathcal{A}, \nu)$ be arbitrary measure spaces. We first show that every isometry $T: L^{p}(X) \rightarrow L^{q}(Y)$ is disjointness preserving for $1 \leq p, q<\infty$, $p, q \neq 2$. Indeed, it is easy to see that $\|f+g\|^{p}+\|f-g\|^{p}=2\left(\|f\|^{p}+\|g\|^{p}\right)$ if and only if $f \cdot g=0$ a.e. $[\mu]$ (ref. [13, p. 416]). Since $T$ is an isometry, for all $f, g$ in $L^{p}(X)$

$$
\begin{aligned}
f \cdot g=0 \text { a.e. }[\mu] & \Leftrightarrow\|f+g\|^{p}+\|f-g\|^{p}=2\left(\|f\|^{p}+\|g\|^{p}\right) \\
& \Leftrightarrow\|T f+T g\|^{q}+\|T f-T g\|^{q}=2\left(\|T f\|^{q}+\|T g\|^{q}\right) \\
& \Leftrightarrow T f \cdot T g=0 \text { a.e. }[\nu] .
\end{aligned}
$$

Hence $T$ is disjointness preserving.

Recall that the function space $L^{\infty}(X)$ is a commutative $C^{*}$-algebra with identity, equipped with the natural algebraic structure and the natural involution. By the Gelfand-Naimark theorem (see, e.g., [4, p. 236], $L^{\infty}(X)$ is isometrically *isomorphic to $C(\Sigma)$, where $\Sigma$ is the maximal ideal space of $L^{\infty}(X)$. Note that $\Sigma$ is compact. 
Let $\Lambda$ be the Gelfand transform from $L^{\infty}(X)$ onto $C(\Sigma)$. We write $\widehat{f}$ for $\Lambda(f)$ for simplicity of notations. For $B$ in $\mathcal{B}$, since $\chi_{B}^{2}=\chi_{B}$, we have $\widehat{\chi B}^{2}=\widehat{\chi_{B}}$. Then $\widehat{\chi_{B}}$ is the characteristic function of a closed and open subset $U_{B}$ of $\Sigma$. Conversely, if $U$ is a closed and open subset of $\Sigma$, then $\chi_{U} \in C(\Sigma)$ and $\chi_{U}=\widehat{f}$ for some $f$ in $L^{\infty}(X)$. Moreover, $\widehat{f^{2}}=\widehat{f}$ and $f^{2}=f$ in $L^{\infty}(X)$. It follows that $f=\chi_{B}$ for some $B$ in $\mathcal{B}$. Consequently, we have

Lemma 1. Every closed and open subset of $\Sigma$ is of the form $U_{B}$ for some $B$ in $\mathcal{B}$.

Let $(X, \mathcal{B}, \mu)$ and $(Y, \mathcal{A}, \nu)$ be measure space, and $\Sigma_{1}$ (resp. $\left.\Sigma_{2}\right)$ the maximal ideal space of $L^{\infty}(X)$ (resp. $L^{\infty}(Y)$ ). For any given map $T: L^{\infty}(X) \rightarrow L^{\infty}(Y)$, define $\widehat{T}: C\left(\Sigma_{1}\right) \rightarrow C\left(\Sigma_{2}\right)$ by $\widehat{T} \widehat{f}=\widehat{T f}$ for all $f$ in $L^{\infty}(X)$. It is clear to get the following proposition.

Proposition 1. $T$ is a bounded linear operator if and only if $\widehat{T}$ is (and $\|T\|=\|\widehat{T}\|) ; T$ is a linear isometry if and only if $\widehat{T}$ is; $T$ is disjointness preserving if and only if $\widehat{T}$ is; and $T$ is invertible if and only if $\widehat{T}$ is (in this case, $\widehat{T}^{-1}=\widehat{T^{-1}}$ ).

Lemma 2. Every surjective linear isometry $T: L^{\infty}(X) \rightarrow L^{\infty}(Y)$ is disjointness preserving.

Proof. It follows Banach-Stone Theorem and the proposition above.

It is plain that there exists a linear isometry $T: L^{\infty}(X) \rightarrow L^{\infty}(Y)$ such that $T$ is not disjointness preserving. (Consider, e.g., $T\left(x_{1}, x_{2}, \ldots,\right)=\left(\frac{x_{1}+x_{2}}{2}, x_{1}, x_{2}, \ldots\right)$ from $\ell^{\infty}$ into $\ell^{\infty}$.)

For the Lamperti problem, Lamperti's proof [10, p. 461] can be modified to prove the following theorem.

Theorem 2. If $(X, \mathcal{B}, \mu)$ is a $\sigma$-finite measure space, $(Y, \mathcal{A}, \nu)$ an arbitrary measure space and $T: L^{p}(X) \rightarrow L^{q}(Y)(1 \leq p, q<\infty$ and $p, q \neq 2)$ a bounded disjointness preserving linear operator, then $T$ is a Lamperti map.

It remains to prove the case $p=q=\infty$ for Lamperti problem. We need the following theorem.

Theorem 3. ([8]) If $X$ and $Y$ are compact Hausdorff space and $T: C(X) \rightarrow$ $C(Y)$ is a surjective disjointness preserving linear operator, then there exists a homeomorphism $\varphi: Y \rightarrow X$ and a function $h$ in $C(Y)$ with $h(y) \neq 0$ for all $y$ in $Y$ such that $T f=h \cdot(f \circ \varphi)$ for all $f$ in $C(X)$.

Theorem 4. Let $(X, \mathcal{B}, \mu)$ and $(Y, \mathcal{A}, \nu)$ be measure spaces. If $T: L^{\infty}(X) \rightarrow$ $L^{\infty}(Y)$ is a bounded surjective disjointness preserving linear operator, then there 
exist a proper regular set homomorphism $\Phi: \mathcal{B} \rightarrow \mathcal{A}$ with $\Phi(X)=Y$ and a function $h$ in $L^{\infty}(Y)$ with $h \neq 0$ a.e. $[\nu]$ such that $T \xi_{B}=h \cdot \xi_{\Phi(B)}$ for all $B$ in $\mathcal{B}$. In other words, $T$ is a Lamperti map.

Proof. Let $\Sigma_{1}$ (resp. $\Sigma_{2}$ ) be the maximal ideal space of $L^{\infty}(X)$ (resp. $L^{\infty}(Y)$ ). Let $\widehat{T}: C\left(\Sigma_{1}\right) \rightarrow C\left(\Sigma_{2}\right)$ be defined by $\widehat{T} \widehat{f}=\widehat{T f}$ for all $f$ in $L^{\infty}(X)$ (via the Gelfand transform $\Lambda$ ). By Proposition $1, \widehat{T}$ is a bounded surjective disjointness preserving linear operator. By Theorem 3, there exist a homeomorphism $\varphi: \Sigma_{2} \rightarrow$ $\Sigma_{1}$ and a function $h$ in $L^{\infty}(X)$ with $h \neq 0$ a.e. $[\nu]$ such that $\widehat{T} \widehat{f}=\widehat{h} \cdot(\widehat{f} \circ \varphi)$ for all $f$ in $L^{\infty}(X)$. Let $\Phi: \mathcal{B} \rightarrow \mathcal{A}$ be defined, modulo null sets, by $\Phi(B)=A$ if $U_{A}=$ $\varphi^{-1}\left(U_{B}\right)$ in the notations of Lemma $1 . \Phi$ is a proper regular set homomorphism.

It is easy to see that $\Phi$ preserves differences and finite unions, and $\nu(\Phi(B))=0$ if and only if $\mu(B)=0$. By the homeomorphism of $\varphi, \Phi$ is surjective and $\Phi(X)=$ $Y$.

It remains to show that $\Phi$ preserves countable union. Suppose that $\left\{B_{n}\right\}_{n}$ is a sequence of measurable sets in $\mathcal{B}$, we need to show that $\Phi\left(\bigcup_{n=1}^{\infty} B_{n}\right)=$ $\bigcup_{n=1}^{\infty} \Phi\left(B_{n}\right)$, or equivalently, $\varphi^{-1}\left(U_{\cup B_{n}}\right)=\bigcup \varphi^{-1}\left(U_{B_{n}}\right)\left(=\sup \varphi^{-1}\left(U_{B_{n}}\right)\right)$. Clearly, $\varphi^{-1}\left(U_{\cup B_{n}}\right)$ is an upper bounded of $\left\{\varphi^{-1}\left(U_{B_{n}}\right)\right\}_{n}$. Suppose that $U_{A}$ is anothre upper bound of $\left\{\varphi^{-1}\left(U_{B_{n}}\right)\right\}_{n}$. Since $\Phi$ is surjective, there is a $B$ in $\mathcal{B}$ such that $\Phi(B)=A$. By assumption, $\varphi^{-1}\left(U_{B_{n}}\right) \subset U_{A}=\varphi^{-1}\left(U_{B}\right)$, then $U_{B_{n}} \subset U_{B}$ for all $n \in \mathbb{N}$. Since $U_{\cup B_{n}}=\sup U_{B_{n}}$, we have $\varphi^{-1}\left(U_{\cup B_{n}}\right) \subset \varphi^{-1}\left(U_{B}\right)=U_{A}$. Therefore, $\varphi^{-1}\left(U_{\cup B_{n}}\right)=\sup \varphi^{-1}\left(U_{B_{n}}\right)$. This establishes the claim.

Finally, observe that, for all $B$ in $\mathcal{B}$,

$$
\begin{aligned}
\widehat{T \chi_{B}} & =\widehat{T} \widehat{\chi_{B}}=\widehat{h} \cdot\left(\widehat{\chi_{B}} \circ \varphi\right)=\widehat{h} \cdot\left(\chi_{U_{B}} \circ \varphi\right)=\widehat{h} \cdot \chi_{\varphi^{-1}\left(U_{B}\right)} \\
& =\widehat{h} \cdot \chi_{U_{\Phi(B)}}=\widehat{h} \cdot \widehat{\chi_{\Phi(B)}}=h \cdot \widehat{h \cdot \chi_{\Phi(B)}} .
\end{aligned}
$$

Hence, $T \chi_{B}=h \cdot \chi_{\Phi(B)}$ for all $B$ in $\mathcal{B}$.

As an immediate consequence of Theorems 2 and 4, we have the following

Theorem 5. Every $\sigma$-finite measure space solves the Lamperti problem.

\section{The Banach-Stone Problem}

In this section, we devote to the Banach-Stone problem. First, let us to see a special case.

Proposition 6. If $1 \leq p, q \leq \infty$, and $T: \ell^{p} \rightarrow \ell^{q}$ is a bounded disjointness preserving operator, then there exist a map $\varphi: \mathbb{N} \rightarrow \mathbb{N}$ and a function $h$ in $\ell^{\infty}$ such that,

$$
T x=h \cdot(x \circ \varphi) \text { for all } x \in \ell^{p} .
$$


Proof. For each $m$ in $\mathbb{N}$, define $e_{m}: \mathbb{N} \rightarrow \mathbb{N}$ by $e_{m}(n)=1$ if $m=n$, and $e_{m}(n)=0$ otherwise. For each $n$ in $\mathbb{N}$, define $\delta_{n}$ to be the linear functional on $\ell^{q}$ sending $e_{n}$ to 1 , and $e_{m}$ to 0 if $m \neq n$. For each $n$ in $\mathbb{N}$, define $\Phi(n)=\{m$ : $\left.T e_{m}(n) \neq 0\right\}$. Since $T$ is disjoint preserving, $\Phi(n)$ contains at most one element.

Let $\mathbf{N}_{0}=\{n: \Phi(n)=\emptyset\}$. It is easy to see that $\left\{n: \delta_{n} \circ T=0\right\} \subset \mathbf{N}_{0}$. On the other hand, let $n \in \mathbf{N}_{0}$. Since the linear span of $\{e m: m \in \mathbb{N}\}$ is weak ${ }^{a}$ st-dense in $\ell^{p}, \delta_{n} \circ T=0$. Hence, $\mathbf{N}_{0}=\left\{n: \delta_{n} \circ T=0\right\}$.

Let $n_{0}$ be a fixed natural number. Define $\varphi: \mathbb{N} \rightarrow \mathbb{N}$ by $\varphi(n)=m$ where $m$ is the unique element in $\Phi(n)$ if $n \in \mathbb{N} \backslash \mathbf{N}_{0}$, and $\varphi(n)=n_{0}$ if $n \in \mathbf{N}_{0}$.

If $n \in \mathbb{N} \backslash \mathbf{N}_{0}$, then there is a scalar $\alpha_{n} \neq 0$ such that $\delta_{n} \circ T=\alpha_{n} \cdot \delta_{\varphi(n)}$. To see this, if $n \in \mathbb{N} \backslash \mathbf{N}_{0}$, then it is easy to see that $\operatorname{ker}\left(\delta_{n} \circ T\right)$ is a nontrivial subspace of $\ell^{p}$. Since $\delta_{n} \circ T$ and $\delta_{\varphi(n)}$ are linear functionals defined on $\ell^{p}$, it suffices to show that $\operatorname{ker}\left(\delta_{n} \circ T\right)=\operatorname{ker} \delta_{\varphi(n)}$. Let $x \in \operatorname{ker} \delta_{\varphi(n)}$, that is, $x(\varphi(n))=0$. Since $T e_{\varphi(n)}(n) \neq 0$ and $T$ is disjointness-preserving, we have $T x(n)=0$, that is, $x \in \operatorname{ker}\left(\delta_{n} \circ T\right)$. That is, $\emptyset \neq \operatorname{ker} \delta_{\varphi(n)} \subset \operatorname{ker}\left(\delta_{n} \circ T\right)$. Notice that $\operatorname{ker} \delta_{\varphi(n)}$ and $\operatorname{ker}\left(\delta_{n} \circ T\right)$ have same codimension. Therefore, $\operatorname{ker}\left(\delta_{n} \circ T\right)=\operatorname{ker} \delta_{\varphi(n)}$. This establishes the claim.

Now, let $h: \mathbb{N} \rightarrow \mathbb{K}$ be defined by $h(n)=\alpha_{n}$ if $n \in \mathbb{N} \backslash \mathbf{N}_{0}$, and $h(n)=0$ otherwise. Then $\delta_{n} \circ T=h(n) \cdot \delta_{\varphi(n)}$ and thus $(T x)(n)=h(n) \cdot x(\varphi(n))$ for all $x$ in $\ell^{p}$ and all natural numbers $n$. Also, $|h(n)|=\left|h(n) \cdot e_{\varphi(n)}(\varphi(n))\right|=\left|T e_{\varphi(n)}(n)\right| \leq$ $\left\|T e_{\varphi(n)}\right\|_{q} \leq\|T\|$ for all natural numbers $n$. Thus $\|h\|_{\infty}=\|T\|$ and this completes the proof.

Now, we consider the Banach-Stone problem in the general case. Observe that the gap between Lamperti map and BS map is the extent to which whethera regular set homomorphism can be induced by a point map. To be more precise, we introduce the following notion.

For a measure space $(X, \mathcal{B}, \mu)$ a member $B$ of $\mathcal{B}$ is an atom of $\mu$ if $\mu(C)=\mu(B)$ or $\mu(C)=0$ for all $C$ in $\mathcal{B}$ with $C \subset B$. We call $(X, \mathcal{B}, \mu)$ an atom-free measure space if $\mu$ posseses no atom. We call a measure subspace $X^{\prime}$ of $X$ a maximal atom free subspace if $X^{\prime}$ is atom-free and $X \backslash X^{\prime}$ is a disjoint union of atoms of $\mu$.

We say that an atom-free measure space $(X, \mathcal{B}, \mu)$ has Sikorski's property if, for an arbitrary measure space $(Y, \mathcal{A}, \nu)$, every regular set homomorphism $\Phi: \mathcal{B} \rightarrow \mathcal{A}$ with $\Phi(X)=Y$ is induced by a measurable point map $\varphi$, that is, $\Phi(B)=\varphi^{-1}(B)$ for any $B$ in $\mathcal{B}$.

Remark. It is known (cf., $\left[13\right.$, p. 397]) that $\left([0,1], \mathcal{B}_{[0,1]}, \mu\right)$ has Sikorski's property, where $\mathcal{B}_{[0,1]}$ is the Borel $\sigma$-algebra and $\mu$ is any $\sigma$-finite regular measure on $\mathcal{B}_{[0,1]}$.

Theorem 7. Every $\sigma$-finite measure space $(X, \mathcal{B}, \mu)$ which has Sikorski's 
property solves the Banach-Stone problem.

Proof. Let $\left\{a_{i}\right\}_{i \in I}$ be an arbitrary maximal set of atoms of $\mu$. Since $\mu$ is $\sigma$ finite, $I$ is at most countable. Let $X_{1}=\bigcup_{i \in I} a_{i}$, then $X_{1}$ works exactly as a subset of $\mathbb{N}$. (For the Banach-Stone problem on $\mathbb{N}$, see Proposition 6.) Thus, without loss of generality, we may assume $\mu$ is atom-free. For an arbitrary measure space $(Y, \mathcal{A}, \nu)$, let $T: L^{p}(X) \rightarrow L^{q}(Y)$ be a (surjective when $p=q=\infty$ ) bounded disjointness preserving operator. It demands to show that $T$ is a BS map. By Theorem 5, there exist a regular set homomorphism $\Phi: \mathcal{B} \rightarrow \mathcal{A}$ and a $h \in L^{q}(Y)$ such that $T \chi_{B}=h \cdot \chi_{\Phi(B)}$ for all $B$ in $\mathcal{B}$ with $\mu(B)<\infty$.

There exists a measurable mapping $\varphi: Y \rightarrow X$ such that $\Phi(B)=\varphi^{-1}(B)$ for all $B$ in $\mathcal{B}$ with $\mu(B)<\infty$. Consequently, $T \chi_{B}=h \cdot\left(\chi_{B} \circ \varphi\right)$.

In case of $p=q=\infty$, it is known that $\Phi(X)=Y$ by Theorem 4. By the Sikorski's property, the claim is done.

For the case, $1 \leq p, q<\infty$ and $p, q \neq 2$, it is not necessarily true that $\Phi(X)=Y$. Let $Y_{0}=\Phi(X)$ be a measurable set. Let $\mathcal{A}_{0}=\left\{Y_{0} \cap A: A \in \mathcal{A}\right\}$ and $\nu_{0}=\left.\nu\right|_{\mathcal{A}_{0}}$. Define $\Phi: \mathcal{B} \rightarrow \mathcal{A}_{0}$ by $\Phi_{0}(B)=Y_{0} \cap \Phi(B)$ for all $B$ in $\mathcal{B}$. It is easy to see that $\Phi_{0}$ is a regualr set homomorphism satisfying $\Phi_{0}(X)=Y_{0}$. By assumption, there exists a measurable mapping $\varphi_{0}: Y_{0} \rightarrow X$ such that $\Phi(B)=\varphi_{0}^{-1}(B)$ for all $B$ in $\mathcal{B}$. It follows that, for all $B$ in $\mathcal{B}$ with $\mu(B)<\infty,\left.\left(T \chi_{B}\right)\right|_{Y_{0}}=\left.\left(h \cdot \chi_{\Phi(B)}\right)\right|_{Y_{0}}=$ $\left.h\right|_{Y_{0}} \cdot \chi_{\Phi_{0}(B)}=h_{0} \cdot \chi_{\varphi^{-1}(B)}=h_{0} \cdot\left(\chi_{B} \circ \varphi_{0}\right)$ where $h_{0}=\left.h\right|_{Y_{0}}: Y_{0} \rightarrow \mathbb{K}$. Since the support of $\left(T \chi_{X}\right)$ is contained in $\Phi(X)=Y_{0}$, we can redefine $h: Y \rightarrow \mathbb{K}$ by $h(y)=h_{0}(y)$ on $Y_{0}$ and $h(y)=0$ otherwise. We can also extend $\varphi_{0}$ to $\varphi: Y \rightarrow X$ by $\varphi(y)=\varphi_{0}(y)$ on $Y_{0}$ and $\varphi(y)=x_{0}$ otherwise for some fixed $x_{0}$ in $X$. Then both $h$ and $\varphi$ are measurable and $T \chi_{B}=h \cdot\left(\chi_{B} \circ \varphi\right)$. This establishes the claim.

Now, if $s$ is any simple function which vanishes outside a set of finite measure, we have $T s=h \cdot(s \circ \varphi)$ by the linearity of $T$. Let $f$ be in $L^{p}(X)$. By passing to a sequence of simple functions which approximate $f$, we have $T f=h \cdot(f \circ \varphi)$. Hence, $T$ is a BS map.

In the following, we shall give a $\sigma$-finite measure space which has Sikorski's property.

Definition 3.1. A totally ordered space $(X, \leq)$ is said to be Dedekind complete if every bounded below nonempty subset has an infimum in $X$. A $\sigma$-algebra $\mathcal{B}$ is called the order $\sigma$-algebra of $X$ if it is generated by all order intervals $(a, b)=$ $\{x \in X: a<x<b\}$. A totally ordered measure space is a totally ordered space with the order $\sigma$-algebra. A measure space $(X, \mathcal{B}, \mu)$ is said to be $\mu$-separable if $(X, \mathcal{B}, \mu)$ is totally ordered and contains a countable subset $D$ of $X$ such that $(a, b) \cap D \neq \emptyset$ for all $a, b$ in $X$ with $\mu((a, b)) \neq 0$. In this case, $D$ is called an order- $\mu$-dense subset of $X$.

Proposition 8. Let $(X, \leq)$ be totally ordered and Dedekind complete. If 
$(X, \mathcal{B}, \mu)$ is a $\sigma$-finite $\mu$-separable measure space, then $(X, \mathcal{B}, \mu)$ has Sikorski's property.

Proof. Let $\left\{a_{i}\right\}_{i \in I}$ be an arbitrary maximal set of atoms of $\mu$. Since $\mu$ is $\sigma$-finite, $I$ is at most countable. Let $X^{\prime}=X \backslash \bigcup_{i \in I} a_{i}$. $X^{\prime}$ is a maximal atom-free measure subspace of $X$. It suffices to show that $X^{\prime}$ has Sikorski's property. Without loss of generality, we may assume $X$ is atom-free.

Given a measure space $(Y, \mathcal{A}, \nu)$ and a regular set homomorphism $\Phi: \mathcal{B} \rightarrow \mathcal{A}$ with $\Phi(X)=Y$. Let $D$ be a countable order- $\mu$-dense subset of $X$. Since $X$ is an atom-free, $\bigcap_{\alpha \in D}(-\infty, \alpha)$ is a null set and $\bigcup_{\alpha \in D}(-\infty, \alpha)=X$. For each $\alpha$ in $D$, define $B_{\alpha}=\Phi(-\infty, \alpha)$. Then $B_{\alpha} \subset B_{\beta}, \alpha \leq \beta$, and $\bigcap_{\alpha \in D} B_{\alpha}=\emptyset$, $\bigcup_{\alpha \in D} B_{\alpha}=Y$. Let $\varphi: Y \rightarrow X$ be defined by $\varphi(y)=\inf \left\{\alpha \in D: y \in B_{\alpha}\right\}$ for all $y$ in $Y$. Since $X$ is (Dedekind) complete, $\varphi$ is well-defined.

For all $x$ in $X$ (we may assume $(-\infty, x) \neq \emptyset$ ), it is easy to see that $\{y \in Y$ : $\varphi(y)<x\}=\bigcup_{\gamma<x, \gamma \in D} B_{\gamma}$. Since $D$ is order- $\mu$-dense in $X$, the set $(-\infty, x) \backslash$ $\bigcup_{\gamma<x, \gamma \in D}(-\infty, \gamma)$ is at most a null set in $X$.

By the facts $\bigcup_{\gamma<x, \gamma \in D}(-\infty, \gamma) \subset(-\infty, x)$ and $\Phi$ is regular set homomorphism, we have that $\bigcup_{\gamma<x, \gamma \in D} B_{\gamma}=\Phi\left(\bigcup_{\gamma<x, \gamma \in D}(-\infty, \gamma)\right)=\Phi(-\infty, x)$. Then $\Phi(-\infty, x)=\{y \in Y: \varphi(y)<x\}=\varphi^{-1}(-\infty, x)$. Therefore, the family $\mathcal{B}^{\prime}=\left\{B \subset X: \Phi(B)=\varphi^{-1}(B)\right\}$ contains all order intervals in $X$. However $\mathcal{B}^{\prime}$ is a $\sigma$-algebra. It follows that $\mathcal{B} \subseteq \mathcal{B}^{\prime}$, i.e., $\Phi(B)=\varphi^{-1}(B)$ for all $B$ in $\mathcal{B}$. And then $\varphi$ is measurable. This complete the proof.

By Theorem 7 and Proposition 8, we get a generalization of Banach's result.

Theorem 9. Every $\sigma$-finite $\mu$-separable measure space $(X, \mathcal{B}, \mu)$, that $(X, \leq)$ is totally ordered and Dedeking complete, solves the Banach-Stone problem.

To end this paper, we give some examples.

Example 10. Let $\mathbb{R}^{n}$ be equipped with usual norm $\|\cdot\|$ and $\mu$ which is Lebesgue measure restricted to the Borel $\sigma$-algebra. Define " $<$ " such that $\mathbf{a}=$ $\left(a_{1}, a_{2}, \ldots, a_{n}\right)<\left(b_{1}, b_{2}, \ldots, b_{n}\right)=\mathbf{b}$ if and only if $\|\mathbf{a}\|<\|\mathbf{b}\|$ or, otherwise, there exists an $i$ such that $a_{j}=b_{j}$ for all $j<i$ and $a_{i}<b_{i}$. Then $\mathbb{R}^{n}$ becomes to be totally ordered and Dedekind complete. Moreover, the $\sigma$-algebra $\mathcal{B}$ generated by all the order intervals induced by $<$ is exactly the usual Borel $\sigma$-algebra for $\mathbb{R}^{n}$. Let $D$ be the set $\left\{\left(d_{1}, d_{2}, \ldots, d_{n}\right) \mid d_{i} \in \mathbb{Q}\right.$ for all $\left.i\right\}$. Then $D$ is countable and order$\mu$-dense in $X$. Thus $X$ is $\mu$-separable. Hence $(X, \mathcal{B}, \mu)$ solves the Banach-Stone problem.

Example 11. For the Hilbert cube (that is, $\left\{x \in l^{2}:\left|x_{n}\right| \leq \frac{1}{n}\right\}$ in norm topology) with usual norm $\|\cdot\|$, define " $<$ " s.t. $\mathbf{a}=\left(a_{1}, a_{2}, \ldots\right)<\left(b_{1}, b_{2}, \ldots\right)=\mathbf{b}$ if and only if $\|\mathbf{a}\|<\|\mathbf{b}\|$ or, otherwise, there exists an $i$ such that $a_{j}=b_{j}$ for all 
$j<i$ and $a_{i}<b_{i}$. Then the Hilbert cube becomes a Dedekind complete totally ordered space and the $\sigma$-algebra $\mathcal{B}$ generated by all the order intervals induced by $<$ is exactly the usual Borel $\sigma$-algebra for Hilbert cube. Let $\mu$ be any $\sigma$-finite measure such that $\mu\left(S_{r}\right)=0$ when $S_{r}$ is a maximal atom-free part of the intersection of $\left\{x \in l^{2}:\|x\|_{2}=r\right\}$ and the Hilber cube. Let $D$ be the set $\left\{\left(d_{1}, d_{2}, \ldots\right) \mid d_{i} \in\right.$ $\mathbb{Q}$ for all $i\}$ together with all atoms. Then $D$ is countable and order- $\mu$-dense in $X$. Thus $X$ is $\mu$-separable. Hence solves the Banach-Stone problem.

Let $(X, \mathcal{B}, \mu)$ be a measure space. In case $p=2$, even though $(X, \mathcal{B}, \mu)$ has Sikorski's property, not every (surjective) linear isometry $T: L^{2}(X) \rightarrow L^{2}(X)$ is a BS map. It may also happen that $T$ is not disjointness preserving and not even a Lamperti map.

Example 12. Consider $X=[-\pi, \pi]$. Let $e_{1}(x)=\frac{1}{\sqrt{2 \pi}}$ and $e_{2 n}(x)=\frac{\cos n x}{\sqrt{\pi}}$, $e_{2 n+1}(x)=\frac{\sin n x}{\sqrt{\pi}}$ for $n=1,2, \ldots$ Let $\left\{p_{1}(x), p_{2}(x), p_{3}(x), \ldots\right\}$ be the collection of Legendre polynomials (they can be easily computed by the Gram-Schmidt precess that, for example, $p_{1}(x)=\frac{1}{\sqrt{2 \pi}}, p_{2}(x)=\sqrt{\frac{3}{2 \pi^{3}}} x$ and $p_{3}(x)=\frac{1}{\ell}\left(x^{2}-\frac{2 \pi^{3}}{3}\right)$, where $\ell=\sqrt{\left.\frac{2 \pi^{5}}{5}-\frac{8 \pi^{6}}{9}+\frac{4 \pi^{7}}{9}\right)}$. Then the two families of functions $\left\{e_{1}(x), e_{2}(x), e_{3}(x)\right.$, $\ldots\}$ and $\left\{p_{1}(x), p_{2}(x), p_{3}(x), \ldots\right\}$ are both orthonormal bases of $L^{2}[-\pi, \pi]$. Let $T: L^{2}[-\pi, \pi] \rightarrow L^{2}[-\pi, \pi]$ be the surjective linear isometry such that $T p_{n}=e_{n}$ for all $n=1,2, \ldots$.

\section{REFERENCES}

1. S. Banach, Theorie des Operations Linéaires, Warszawa, 1932.

2. E. Behrends, M-structure and the Banach-Stone Theorem, Lecture Notes in Math., Vol. 736, Berlin-Heidelberg-New York, Springer, 1979.

3. M. Cambern, On $L^{1}$ isomorphisms, Proc. Amer. Math. Soc., 78 (1980), 227-228.

4. J. B. Conway, A Course in Functional Analysis, 2nd ed., Graduate Texts in Math., Vol. 96, Berlin-Heidelberg-New York, Springer, 1990.

5. T. W. Gamlin, Uniform algebras, Englewood Cliffs, N. J., Prentice-Hall, 1969.

6. P. R. Halmos, Measure theory, Graduate Texts in Math., Vol. 18, Berlin-HeidelbergNew York, Springer, 1974.

7. W. Holsztynski, Continuous mappings induced by isometries of spaces of continuous function, Studia Math., 26 (1966), 133-136.

8. K. Jarosz, Automatic continuity of separating linear isomorphisms, Canad. Math. Bull., 33 (1990), 139-144.

9. J.-S. Jeang and N.-C. Wong, Weighted composition operators of $C_{0}(X)$ 's, J. Math. Anal. Appl., 201 (1996), 981-993. 
10. J. Lamperti, On the isometries of certain function-spaces, Pacific J. Math., 8 (1958), 459-466.

11. F. Lessard, Linear operators induced by point transformations, Ann. Sci. Math. Québec, 18(1) (1994), 79-93.

12. W. A. J. Luxemburg and A. C. Zaanen, Riesz Spaces I, Amsterdam, North Holland, 1971.

13. H. L. Royden, Real Analysis, 3rd ed., New York, Macmillan, 1988.

14. R. Schneider, Unit preserving isometries are homomorphisms in certain $L^{p}$, Can. J. Math., 27 (1975), 133-137.

15. A. R. Sourour, The isometries of $L^{p}(\Omega, X)$, J. Funct. Anal., 30 (1978), 276-285.

\author{
Chun-Yen Chou \\ Department of Mathematics Education, \\ National Hualian University of Education, \\ Hualian 970, Taiwan, R.O.C. \\ E-mail: choucy@mail.nhlue.edu.tw \\ Wu-Lang Day \\ National I-Lan Senior High School, \\ Yilan City, Yilan 260, Taiwan, R.O.C. \\ E-mail: yta@mail.itsh.ilc.edu.tw \\ Jyh-Shyang Jeang \\ Department of Management Sciences, \\ 1, Wei-Wu Road, \\ Military Academy, \\ Fengshan, Kaohsiung 830, Taiwan, R.O.C. \\ E-mail: jeangjs@cc.cma.edu.tw
}

